\title{
BIOSTRATIGRAPHIE DES FORAMINIFERES ET INTERPRETATION PALEOENVIRONNEMENTALE DU PUITS OPOLO- I DANS LE DELTA DU NIGER, NIGERIA
}

\author{
Cheoh, J.M ${ }^{1}$, Bassey, C.E ${ }^{2}$, Agyingi, C.M ${ }^{3}$
}

\begin{abstract}
'Département des Sciences de la Terre, Faculté de Science, B.P. 67 Dschang, Cameroun.
${ }^{2}$ Département de Géologie, Faculté des Sciences, Université d'Ibadan, Ibadan, Nigeria.

${ }^{3}$ Département de Géologie et Sciences de l’Environnement, Université de Buea, Cameroun.
\end{abstract}

\begin{abstract}
ARESUME:- 115 déblais de sondage et des logs éléctriques ont été soumis aux analyses biostratigraphiques et paléoenvironnementales pour déterminer la lithologie, l'âge, et l'environnement de dépôt des sédiments provenant du puits Opolo-1 dans le Delta du Niger, Nigeria. Les résultats montre que du bas vers le haut de l'intervalle, les sédiments sont des argiles noires qui passent progresssivement aux sables argileux et finallement au grés au sommet du puits. Les grès sont plus ou moins bien triés, anguleux à subanguleux, fins à grossiers. La présence de la pyrite diagénétique dans toute la section étudiée nous amenent à conclure que le processus de dépôt a eu lieu dans des conditions calmes et réductrices. 124 espèces de foraminifères comprenant 82 benthiques (66\%) et 42 planctiques (34\%) ont été identifiées et utilisées pour la datation, la biozonation et l'interpretation paléoenvironnemental. L'âge du Miocène moyen au Pliocène inférieur est proposée. Sur la base du premier niveau d'apparition des foraminifères planctiques diagnostiques, six biozones ont été reconnues. Les paléoenvironnements de dépôts varient du néritique au bathyal et sont caractérisés par la présence d'espèces tel que Ammobacculites sp., Ammonia beccarii, Quinqueloculina lamarkiana dans les milieux néritiques et Bolivina scalprata miocenica, Cyclammina cancellata, et Heterolepa pseudoungeriana dans les zones bathyales.
\end{abstract}

\section{INTRODUCTION}

Le puits Opolo- 1 est situé sur la longitude $4^{\circ} 80^{\prime}$ E et la latitude $5^{\circ} 43^{\prime} \mathrm{N}$ dans la partie occidentale de l'offshore du Delta du Niger (Fig. 1). Ce puits a une profondeur totale de 3429 m. Grâce à l'avancement rapid des méthodes analytiques moderne utilisée actuellement en géologie du pétrole, et considerant les coûts d'exploration toujours gallopants, les sociétés pétroliéres ont tendance à rentrer dans les puits qui ont été considérés comme non-productif ou marginal. C'est dans cette optique de re-évaluation qu'une étude biostsratigraphique haute - résolution a été initiée pour faire une prospection plus approfondie sur le puits Opolo-1.

Les objectifs de cette étude sont donc basés sur l'identification lithologique, la datation des sédiments, l'intérpretation des milieux de dépôts et la production des données biostratigraphiques nécessaires pour une étude de stratigraphie séquentielle ultérieure. Ces analyses vont augmenter considérablement les possibilités de localisation des roches réservoirs, roches mères et pièges pour les hydrocarbures surtout dans les domaines à hauts risques comme l'offshore.

\section{CONTEXTEGEOLOGIQUE}

Le Delta du Niger est d'âge Cénozoique. C'est le produit d'un troisième et dernier cycle de dépôts qui est une phase essentiellement régressive commencée pendant le Tertiaire inférieur. Trois unités lithostratigraphiques constituées d'une forte séquence diachronique (Eocène - Récent) ont été reconnues en surface et subsurface du Delta du Niger (Short et Stauble,1967). Du bas vers le haut, ce sont: La formation d'Akata, la formation d'Agbada, et la formation de Benin. La formation d'Akata est composée d'argiles de pro-delta déposées sur le talus continental dans les eaux profondes. Pour la formation d'Agbada, elle est une 


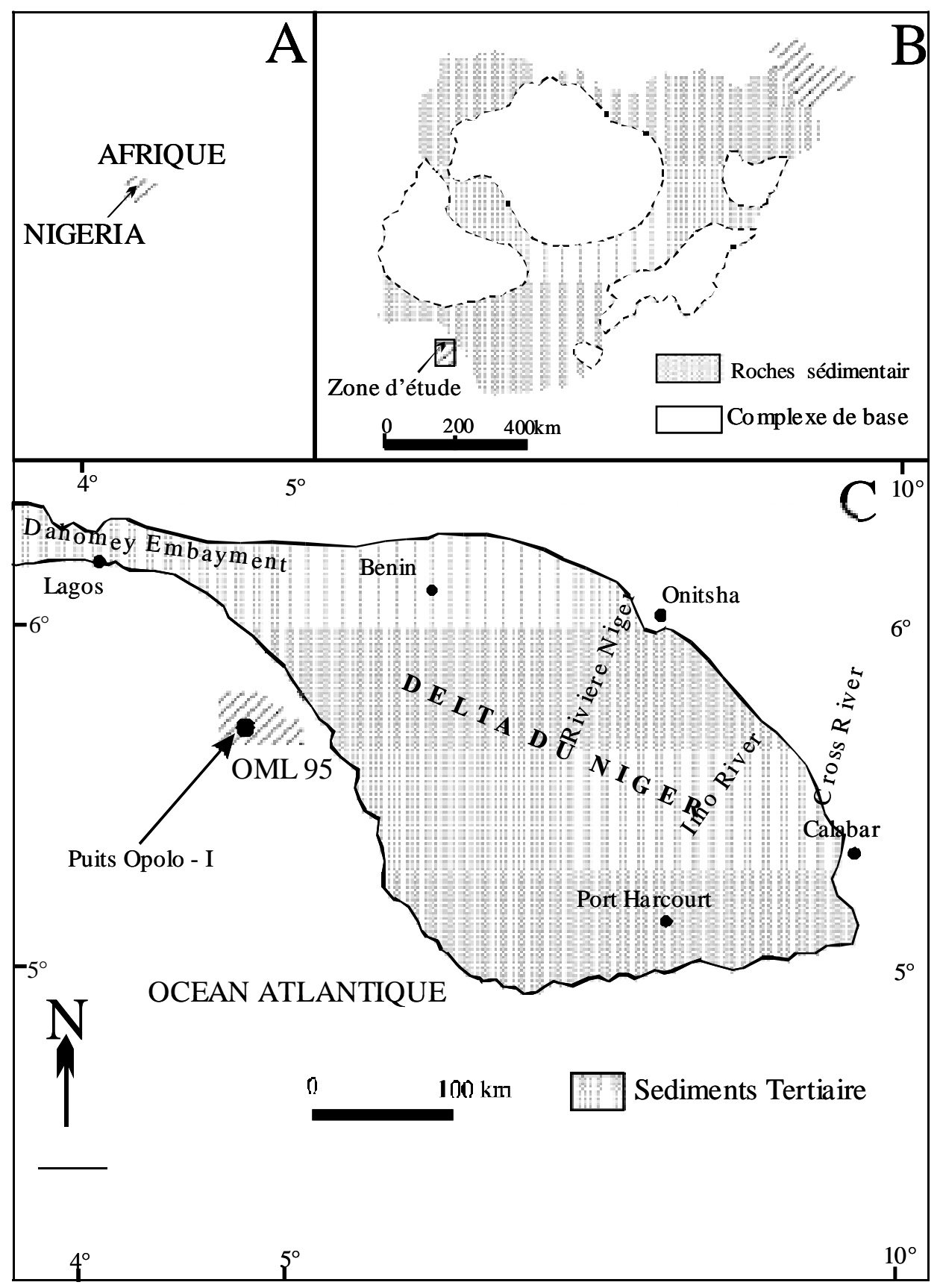

Fig.1: Carte de localisation du puits Opolo-1, Delta du Niger 
séquence paralique de grès et d'argiles intercalés, déposés dans les milieux néritiques peu profond. Les unités de sables dans la formation d'Agbada constituent les roches réservoirs pour le pétrole tandis que les argiles fonctionnent comme roches méres et pièges ( Short et Stauble, 1967). La formation de Benin est composée des sables fluviatiles déposés en milieu continental (Weber, 1971). Conventionnellement, la base de la formation de Benin est reconnue par la première apparition des fossiles strictement marin.

\section{METHODOLOGIE}

Cette étude a été éffectuée sur 115 déblais de sondages et des logs éléctriques (Gamma Ray et SP) provenant du puits Opolo-1. Opolo-1 a une profondeur totale de $3429 \mathrm{~m}$ et les échantillons ont été collectés à intervalle de $10 \mathrm{~m} .10$ g de chaque échantillon sont soumis aux méthodes standards paléontologiques pour la récolte des foraminifères par lavage et tamisage. Les foraminifères et d'autres microfaunes ont été dégagés du résidus seché à partir d'un tamis à mailles de $65 \mu$ utilisant un microscope binoculaire de marque Leitz Wetzler. Les foraminifères dégagés ont été préservés dans les lames micropaléontologiques numérotés pour l'identification et les analyses statistiques.

\section{LITHOSTRATIGRAPHIE}

Une description lithologique des déblais de sondage a été intégrée avec l'examen des logs Gamma et l'on constate que l'intervalle pénétré appartient à la formation d'Agbada (Fig. 2). La section est donc divisée en deux unités:

- unité inférieure essentiellement argileux avec une épaisseur stratigraphique de 1387m (3429 - 2042 m) qui devient de plus en plus gréseux vers le haut;

- $\quad$ unité supérieure dominée par les sables fin, anguleux à subanguleux, plus ou moins bien trié alternant avec des argiles minces; épaissseur 762 m (2042 - 1280 $\mathrm{m})$.

On note ici que cette augmentation en quantité d'argile avec la profondeur à était déjâ signalée par Short et Stauble (1967) pour la formation d'Agbada. Les argiles sont gris noire à noire, massives avec quelques petites intercalations de sables. La pyrite est ubiquite dans presque tous les échantillons analysés.

\section{BIOSTRATIGRAPHIE}

\section{Détermination de l’âge des sédiments}

124 espèces des foraminifères comprenant 82 benthiques (66\%) et 42 planctiques ont été idéntifiées et utilisées pour la datation, la biozonation et l'interpretation paléoenvironnementale. Sur la base des différentes espèces des foraminifères récoltés du puits Opolo-1, et à partir de la répartition stratigraphique de quelques espèces planctiques diagnostiques utilisés par Berggren (1969) et Mboro et al., (1981), l'âge de Miocène moyen au Pliocène inférieur est proposé pour les sédiments.

\section{Miocène moyen}

L'intervalle du puits répresentant le Miocène moyen varie de 3190 à $2980 \mathrm{~m}$. On ne pouvait par définir avec précision la base du Miocène moyen, mais le niveau de la première apparition (First Appearance Datum, FAD) d'Orbulina universa, une espèce importante marquant la limite Miocène inférieur - Miocène moyen à une profondeur de $3190 \mathrm{~m}$, peut être considérée comme la base du Miocène moyen ( Mboro et al.,1981). Le sommet de l'intervalle est marqué par la première apparition de Globorotalia acostaensis qui est une espèce importante délimitant le Miocène moyen (Fig. 3). Les autres foraminifères planctiques caractéristiques qui ont été trouvées dans cette intervalle sont Globorotalia peripheroronda, $G$. menardii, Globigerina nepenthes et Praeorbulina glomerosa.

\section{Pliocène inférieur}

Cet intervalle a une épaisseur stratigraphique de $440 \mathrm{~m}$ $(1720$ - 1280 m). La base du Pliocène inférieur est indiquée par le niveau de première apparition de Globorotalia margaritae à une profondeur de $1720 \mathrm{~m}$ qui correspond à la limite entre le Miocène et le Pliocène. Le sommet du Pliocène inférieur ne pouvait par être définit dans Opolo 1 à cause de l'absence des foraminifères planctiques plus jeunes que le Pliocène inférieur. Cita (1973) a signalé l'apparition initiale de G. margaritae à la limite Miocène Pliocène dans les sédiments de la Méditerannee, et Parker (1973) a utiliser $G$. margaritae comme un marqueur du Pliocène inférieur. Stainforth et al., (1975) et Keller (1979) ont aussi utilisé le niveau de prémière apparition de $G$. margaritae pour définir la limite inférieur du Pliocène dans différentes régions. En conséquence, la limite inférieure 


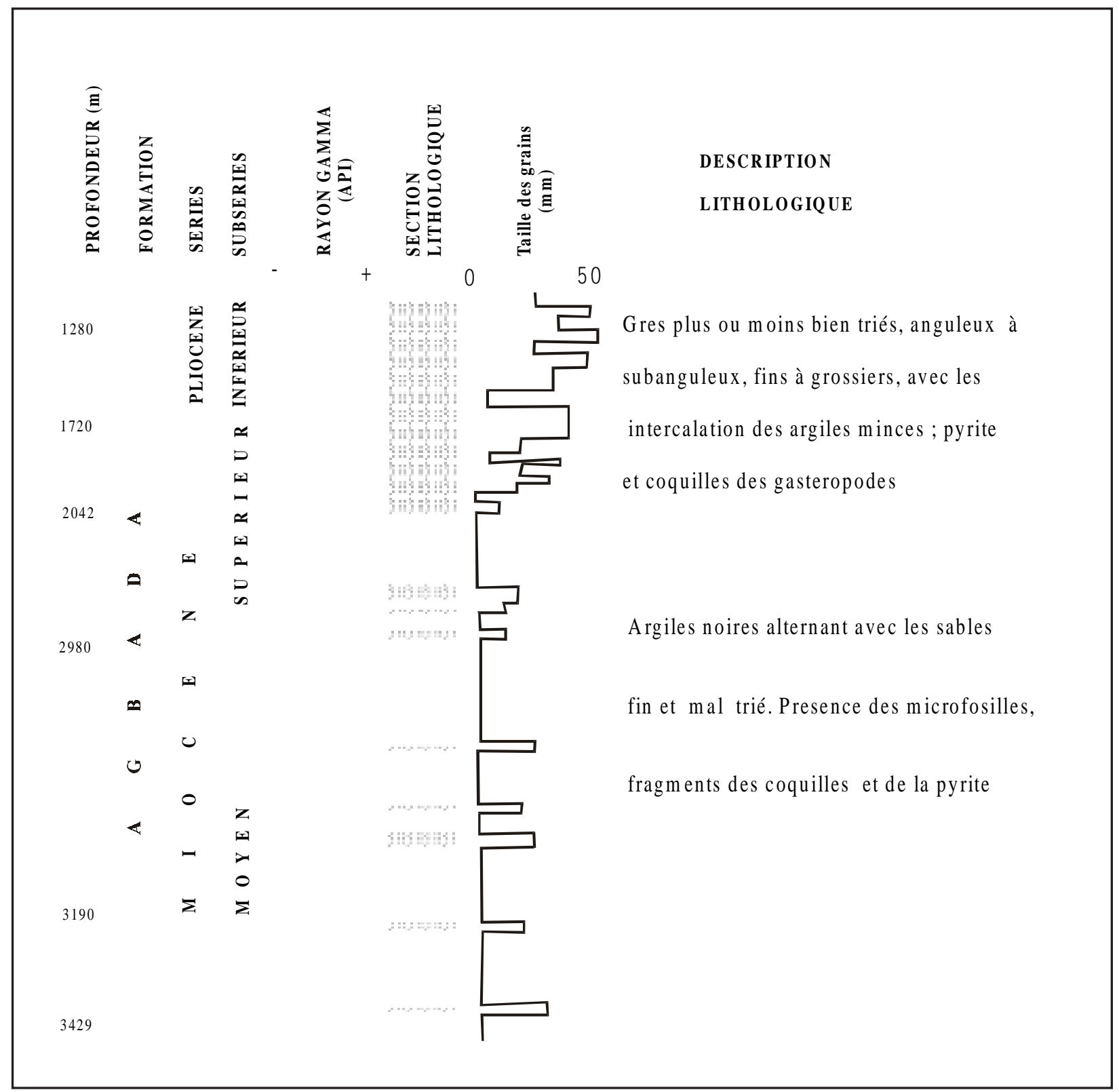

Figure 2: Age et lithologie des sédiments dans le puits Opolo I, Delta du Niger 


\begin{tabular}{|c|c|c|c|c|c|c|c|}
\hline AGE & $\begin{array}{l}\text { BOLLI, } \\
1966\end{array}$ & $\begin{array}{l}\text { BLOW } \\
1969\end{array}$ & $\begin{array}{l}\text { POSTUMA, } \\
1971\end{array}$ & $\begin{array}{l}\text { MBORO et al. } \\
1981\end{array}$ & $\begin{array}{l}\text { PROPOSEE } \\
\text { DANS CET } \\
\text { ETUDE }\end{array}$ & ZONE & 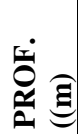 \\
\hline $\begin{array}{l}\text { PLIOCENE } \\
\text { INFERIEUR }\end{array}$ & $\begin{array}{l}\text { Globorotalia } \\
\text { margaritae }\end{array}$ & $\begin{array}{l}\text { Globorotalia tumida } \\
\text { tumida- } \\
\text { Spaeroidinellopsis } \\
\text { subdehiscens } \\
\text { paenedehiscence }\end{array}$ & $\begin{array}{l}\text { Globorotalia } \\
\text { margaritae }\end{array}$ & $\begin{array}{l}\text { Globorotalia } \\
\text { crassaformis }\end{array}$ & $\begin{array}{l}\text { Globorotalia } \\
\text { margaritae }\end{array}$ & N18 & \multirow[t]{2}{*}{$\begin{array}{l}1280 \\
1720\end{array}$} \\
\hline $\begin{array}{l}\text { MIOCENE } \\
\text { SUPERIEUR }\end{array}$ & $\begin{array}{l}\text { Globorotalia } \\
\text { dutertrei }\end{array}$ & $\begin{array}{l}\text { Globorotalia } \\
\text { tumida plesiotumida }\end{array}$ & $\begin{array}{l}\text { Globorotalia } \\
\text { dutertrei }\end{array}$ & $\begin{array}{l}\text { Globorotalia } \\
\text { tumidia } \\
\text { plesiotumida }\end{array}$ & $\begin{array}{l}\text { Globorotalia } \\
\text { merotumida }\end{array}$ & N17 & \\
\hline TORTONIEN & $\begin{array}{l}\text { Globorotalia } \\
\text { acostaensis }\end{array}$ & $\begin{array}{l}\text { Globorotalia } \\
\text { acostaensis } \\
\text { acostaensis- } \\
\text { Globorotalia } \\
\text { merotumida }\end{array}$ & $\begin{array}{l}\text { Globorotalia } \\
\text { acostaensis }\end{array}$ & $\begin{array}{l}\text { Globorotalia } \\
\text { acostaensis }\end{array}$ & $\begin{array}{l}\text { Globorotalia } \\
\text { acostaensis }\end{array}$ & N16 & 2670 \\
\hline \multirow{3}{*}{$\begin{array}{l}\text { MIOCENE } \\
\text { MOYEN }\end{array}$} & $\begin{array}{l}\text { Globorotalia } \\
\text { menardii }\end{array}$ & $\begin{array}{l}\text { Globorotalia } \\
\text { continuosa }\end{array}$ & $\begin{array}{l}\text { Globorotalia } \\
\text { menardii }\end{array}$ & $\begin{array}{l}\text { Globorotalia } \\
\text { menardii }\end{array}$ & $\begin{array}{l}\text { Globorotalia } \\
\text { menardii }\end{array}$ & N15 & \multirow{3}{*}{$\begin{array}{l}3005 \\
3130\end{array}$} \\
\hline & $\begin{array}{l}\text { Globorotalia } \\
\text { mayeri }\end{array}$ & $\begin{array}{l}\text { Globigerina nepenthes } \\
\text {-Globorotalia } \\
\text { siakensis }\end{array}$ & $\begin{array}{l}\text { Globorotalia } \\
\text { siakensis }\end{array}$ & $\begin{array}{l}\text { Globorotalia } \\
\text { mayeri }\end{array}$ & $\begin{array}{l}\text { Globigerina } \\
\text { nepenthes }\end{array}$ & N14 & \\
\hline & $\begin{array}{l}\text { Globorotalia } \\
\text { fohsi } \\
\text { barisanensis }\end{array}$ & $\begin{array}{l}\text { Orbulina surturalis - } \\
\text { Globorotalia } \\
\text { peripheroronda }\end{array}$ & $\begin{array}{l}\text { Globorotalia } \\
\text { peripheroronda }\end{array}$ & $\begin{array}{l}\text { Globorotalia } \\
\text { peripheroronda } \\
\text {-Orbulina } \\
\text { suturalis }\end{array}$ & $\begin{array}{l}\text { Globorotalia } \\
\text { peripheroronda }\end{array}$ & N9 & \\
\hline
\end{tabular}

Figure 3 : Corrélation des zones établies dans le puits Opolo -I, Delta du Niger

du Pliocène dans le puits Opolo -1 est placée à une profondeur de $1720 \mathrm{~m}$ qui matérialise le niveau de première apparition de Globorotalia margarita .

\section{LA BIOZONATION}

Six biozones définis par les foraminifères planctiques ont été établies pour les sédiments d'âge Miocène moyen Pliocène inférieur. Ces zones ont été reconnues sur la base des niveaux de la première apparition des foraminifères chronostratigraphiques qui marquent leur limite inférieure, tandis que le niveau de la première apparition des espèces supra-adjacentes marque le sommet de chaque zone (Fig. 3).

Cette méthode a été appliquée au lieu de la première apparition et de la denière apparition (niveau d'extinction) de chaque espèce marqueur pour éviter le problème de contamination résultant de l'effondrement qui est très commun avec les déblais de forage. Toutes ces zones ont été corrélées avec les zones standards de foraminifères planctiques établies par Bolli (1966), Blow (1969), Postuma (1971) et Mboro et al., (1981), et elles sont définie de la base au sommet comme suit:

\section{MIOCENEMOYEN}

\section{(i) Zone à Globorotalia peripheroronda (N9):}

Intervalle stratigraphique: 3190 - $3130 \mathrm{~m}$

Age: Inférieur du Miocène moyen (Helvétien ).

Caractéristiques et corrélation: Le sommet de cette zone est défini par le niveau de la première apparition de Praeorbulina glomerosa à une profondeur de $3130 \mathrm{~m}$, tandis que la base est marquée par la première apparition d'Orbulina universa à une profondeur de $3190 \mathrm{~m}$. Cette zone est caractérisée par la présence de G. peripheroronda, G. Obesa, Orbulina universa et Globigerinoides trilobus. La zone à Globorotalia peripheroronda est équivalente 
à la zone N9 de Blow (1969) et la partie supérieure de la zone à Bolivina éburnensis congolensis - Bolivina imperatrix - Eponides eshira de Mboro et al., (1981) dans le bassin du Congo.

\section{(ii) Zone à Globigerina nepenthes (N14):}

Intervalle stratigraphique: $3080-3005 \mathrm{~m}$.

Age: Miocène moyen.

Caractéristiques et corrélation: La base de la zone à Globigerina nepenthes est délimitée par le niveau de première apparition de Globigerina nepenthes et le sommet défini par la première apparition de Globorotalia menardii à une profondeur de $3005 \mathrm{~m}$. Elle est corrélable avec la zone à Globorotalia mayeri (N14) de Bolli (1966) et Mboro et al., (1981) et aussi avec la zone à Globigerina nepenthes - Globorotalia siakensis de Blow (1969) et Postuma (1971).

\section{Zone à Globorotalia menardii (N15):}

Intervalle stratigraphique: $3005-2980 \mathrm{~m}$.

Age: Partie supérieure du Miocène moyen.

Caractéristiques et corrélation: La base et le sommet de la zone à Globorotalia menardii est indiquée par les niveaux de prémière apparition de Globorotalia menardii et $G$. acostaensis respectivement. Cette zone est caractérisée par la présence d'Orbulina universa et Globigerinoides obliquus. Elle est corrélable à la zone à G. menardii (N15) de Bolli (1966), Postuma (1971), Mboro et al., (1981) et la zone à Globorotalia continuosa (N15) de Blow (1969) (Fig. 3).

\section{MIOCENESUPERIEUR}

\section{(iii) Zone à Globorotalia acostaensis (N16):}

Intervalle stratigraphique: 2980 - $2670 \mathrm{~m}$

Age: Tortonien (partie inférieur du Miocène supérieur).

Caractéristiques et corrélation: La base du Miocène supérieur a été délimitée à une profondeur de 2980 m par la première apparition de $G$. acostaensis et son sommet par l'apparition de G. merotumida à une profondeur de $2670 \mathrm{~m}$. Les autres espèces planctiques de longue durée qui existent avec $G$. acostaensis dans cette zone sont Orbulina universa, O. bilobata et Globigerinoides trilobus. La zone à G. acostaensis est corrélable à la zone à $G$. acostaensis (N16) de Bolli (1966), Postuma (1971) et Mboro et al., (1981).

\section{(iv) Zone à Globorotalia merotumida (N17):}

Intervalle stratigraphique: 2670 - $1720 \mathrm{~m}$

Age: Miocène supérieur

Caractéristiques et corrélation: Le niveau de première apparition de G. merotumida se trouve à une profondeur de $2670 \mathrm{~m}$ marquant ainsi la base de cette zone. $G$. margaritae fait sa première apparition à $1720 \mathrm{~m}$ de profondeur qui est la limite supérieure de la zone à $G$. merotumida. Cette zone est équivalente à la zone à $G$. tumida plesiotumida (N17) de Mboro et al ., (1981) et elle est caractérisée par la présence de G. obesa et Globigerinoides trilobus . A partir de la zone N16 à la zone N17, c'est le Miocène supérieur qui couvre une intervalle stratigraphique allant de $2980 \mathrm{~m}$ à $1720 \mathrm{~m}$. Cet âge est assigné à base de niveau de la première apparition de Globorotalia acostaensis $(2980 \mathrm{~m})$ et Globorotalia margaritae (1720 m). Ciffeli et Glaçon (1979) ont utilisés le niveau de première apparition de $G$. margaritae comme marqueur pour la limite Miocène supérieur - Pliocène inférieur dans l'Atlantique du Nord. Dans l'intervalle du Miocène supérieur, on a aussi trouvé des espèces tel que Globorotalia merotumida, Globigerinoides obliquus, $G$. trilobus et G. sacculifer.

\section{LALIMITEMIOCENE-PLIOCENE}

Dans le puits Opolo -1, la limite Miocène - Pliocène est rencontrée à une profondeur de $1720 \mathrm{~m}$. Cette limite est définie par le niveau de la première apparition de Globorotalia margaritae et elle est considérée comme une limite biostratigraphique très importante pour séparer le Miocène du Pliocène. Ainsi, plusieurs auteurs l'ont utilisée pour cette coupure. C'est le cas dans le site 139 du Projet de forage sous marin (Deep Sea Drilling Project, DSDP) dans l'Atlantique Nord, Stainforth et al., (1975) et Ciffeli et Glaçon (1979) ont utilisés le niveau de première apparition de $G$.margaritae pour délimiter le Miocène supérieur du Pliocène inférieur dans le site 116 de l'Atlantique Nord. A ce niveau, on constate un remarquable changement dans la composition des espèces surtout en ce qui concerne les foraminifères benthiques. Il y a l'absence totale des benthiques arénacés dans les sédiments plus jeunes que le Miocène supérieur. Cette observation peut être expliquée par l'instabilité des tests inorganiques dans l'eau froide qui vraisemblement est le résultat de l'évenement réfroidissant qui a caractérisé le Miocène supérieur (Messinien). Au contraire, on a trouvé quelques foraminifères benthiques à test calcaire au dessus et en dessous de la limite Miocène - Pliocène qui suggère que les tests calcaires sont plus stables dans 
l'eau froide que les tests arénacés inorganiques. Les foraminifères benthiques à tests calcaires idéntifiés de chaque côté de la limite Miocène - Pliocène sont Lenticulina inornata, Marginulina costata, Quinqueloculina lamarkiana, Heterolepa pseudoungeriana et Ammonia beccarii. On propose donc, que les conditions environnementales très défavorables liées aux basses témperatures résultants d'événement refroidissant pendant le Messinien (Miocène supérieur) ont affécté la radiation évolutionnaire des tests inorganiques.

\section{PLIOCENE}

(v) Zone à Globorotalia margaritae (N18):

Intervalle stratigraphique: 1720 - $1280 \mathrm{~m}$

Age: Pliocène inférieur

Caractéristiques et corrélation: La zone à G. margaritae est définie à la base par le niveau de première apparition de G. margaritae qui est un marqueur très important pour délimiter le Miocène du Pliocène. Cette limite se trouve à une profondeur de $1720 \mathrm{~m}$. Le sommet de la zone N18 ne pouvait pas se définir dans le puits Opolo -1 dû à l'absence des éspèces foraminifères planctiques diagnostiques plus jeunes que le Pliocène inférieur. La zone est corrélé avec la zone à Globorotalia crassaformis de Mboro et al., (1981) dans le bassin du Congo.

\section{LESPALEOENVIRONNEMENTSDEDEPOTS}

Quatre méthodes indépendantes mais mutuellement corroborés ont fournies les données qui nous ont permis de déduire les conditions de dépôt des sédiments pendant la tranche stratigraphique Miocène moyen - Pliocène inférieur:

- tendances dans l'abondance et diversités des espèces,

- $\quad$ rapports Planctiques / Benthiques, (P/B),

- $\quad$ rapports entre les différents types des tests pour trois sous ordres benthiques: Textulariina, Miliolina et Rotaliina et

- $\quad$ rapports des tests Calcaires et Arénacés, (C/A).

Les résultats de ces analyses ont été intégrés avec l'étude des caractéristiques lithologiques des sédiments et les signatures sur les Gamma logs. Les environnements de dépôts varient d'une mer peu profonde (marginale) au néritique externe/bathyal avec l'influence directe de la mer (Fig. 4). Un modèle paléoenvironnemental de dépôt avec quelques espèces bathymetriques est proposé (Fig. 5).

\section{ENVIRONNEMENTMARINMARGINAL-NERITIQUE INTERNE}

$$
(1280-1810 \mathrm{~m})
$$

L'intervalle est caractérisé par une faible teneur en abondance et diversités des espèces. Les faibles valeurs en $\mathrm{P} / \mathrm{B}$ et $\mathrm{C} / \mathrm{A}$ ont été énregistrées également. Ces résutats montrent les environnements de dépôts marin peu profond ou marginal. Cette intérpretation est corroborée par la présence des fragments de gasteropodes et lamellibranches (Petters, 1978). La couleur noire des argiles et la présence de la pyrite diagénétique sont des témoins d'un dépôt dans les conditions calmes et réductrices ou anoxiques (Massala et al., 1996). A partir des rapports des différents types des tests pour les sous ordres Textulariina, Miliolina et Rotaliina (Fig. 6), les conditions hyposalines marécageuses et lagonaux avec une salinité de moins de $35 \%$ est proposée (Murray,1973). Les foraminifères benthiques d'une mer peu profonde à test calcaire retrouvés dans cet intervalle sont Lenticulina inornata, Marginulina costata, Ammonia beccarii, Quinqueloculina lamarkiana, Bolivina scalprata miocenica et Eponides eshira.

\section{ENVIRONNEMENTNERITIQUEMOYEN-NERITIQUE EXTERNE}

$$
(1810-2505 \mathrm{~m})
$$

Pendant le Miocène supérieur, le niveau de la mer semblait avoir progressivement augmenté dû probablement à un phénomène de transgression. Cette observation est mise en evidence par une remarquable accroissement du nombre et de la diversité des espèces foraminfères. On note aussi qu'il y a une augmentation dans les rapports $\mathrm{P} / \mathrm{B}$. Pendant cette période, les formes benthiques ont dominées les communautés indigénes de la faune comprenant les espèces tels que Cyclammina cancellata, Haplophragmoides compressa et Alveolophragmium crassum. Massala et al., (1996) ont montré qu'une augmentation des rapports $\mathrm{P} / \mathrm{B}$ est expliquée par une augmentation de la mer ou par un changement dans les conditions de dépôt au fond de la mer. Dans cette étude, on a remarqué un mélange entre les espèces d'eau peu profonde comme Lenticulina inornata et Ammonia beccarii avec les assemblages qui sont souvent limités dans les eaux profondes. Une telle association peut être considerée comme l'effet d'un transport post - mortem des espèces vivants dans l'eau peu profonde en milieu profonds ou simplement par les effets de bioturbation (Mboro et al.,1981). Néanmoins, une autre preuve qui confirme l'environnement de dépôt proposé pour cet 


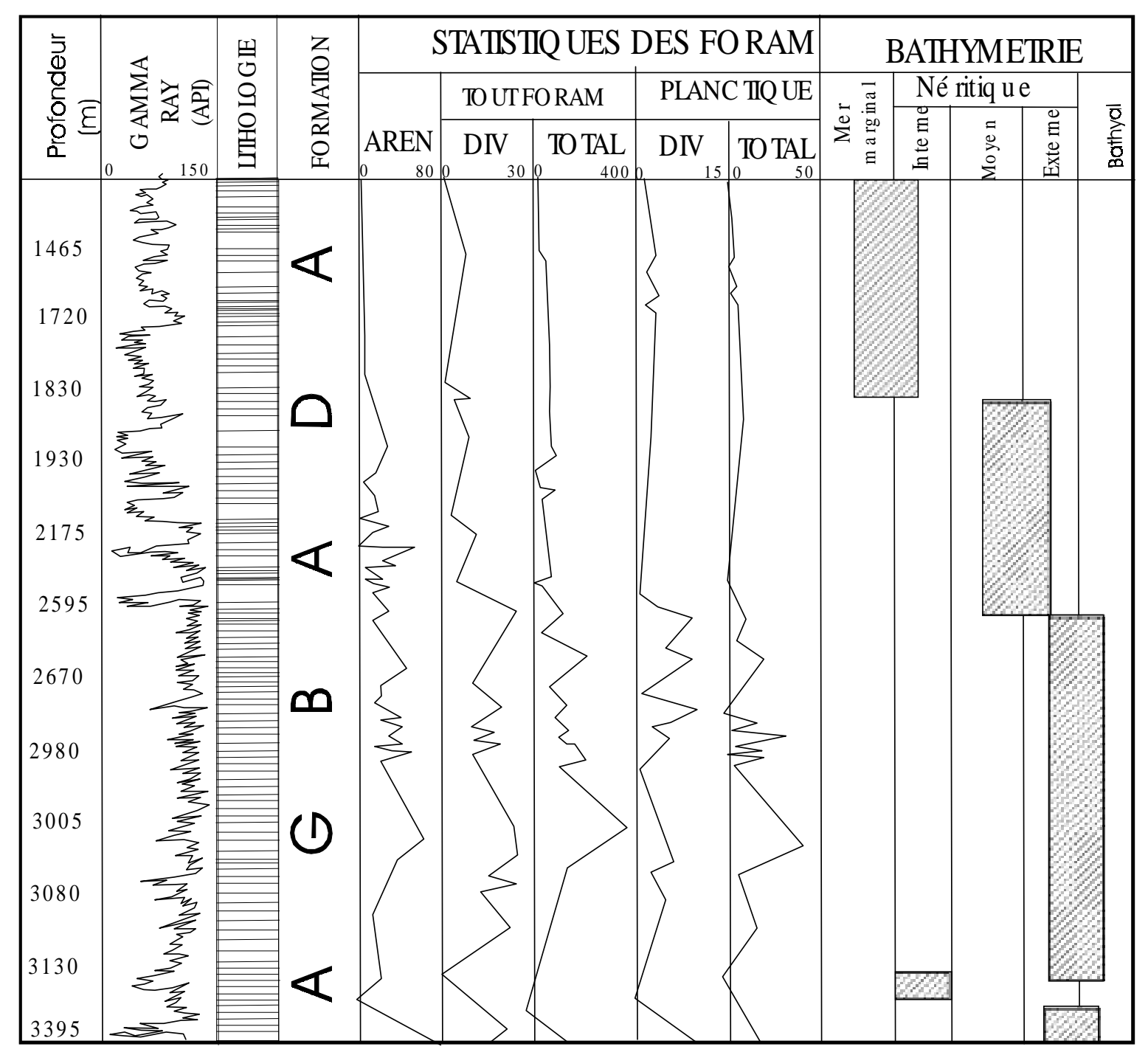

Figure 4: Analyse statistique et biostratigraphique du puits Opolo -1, Delta du Niger 


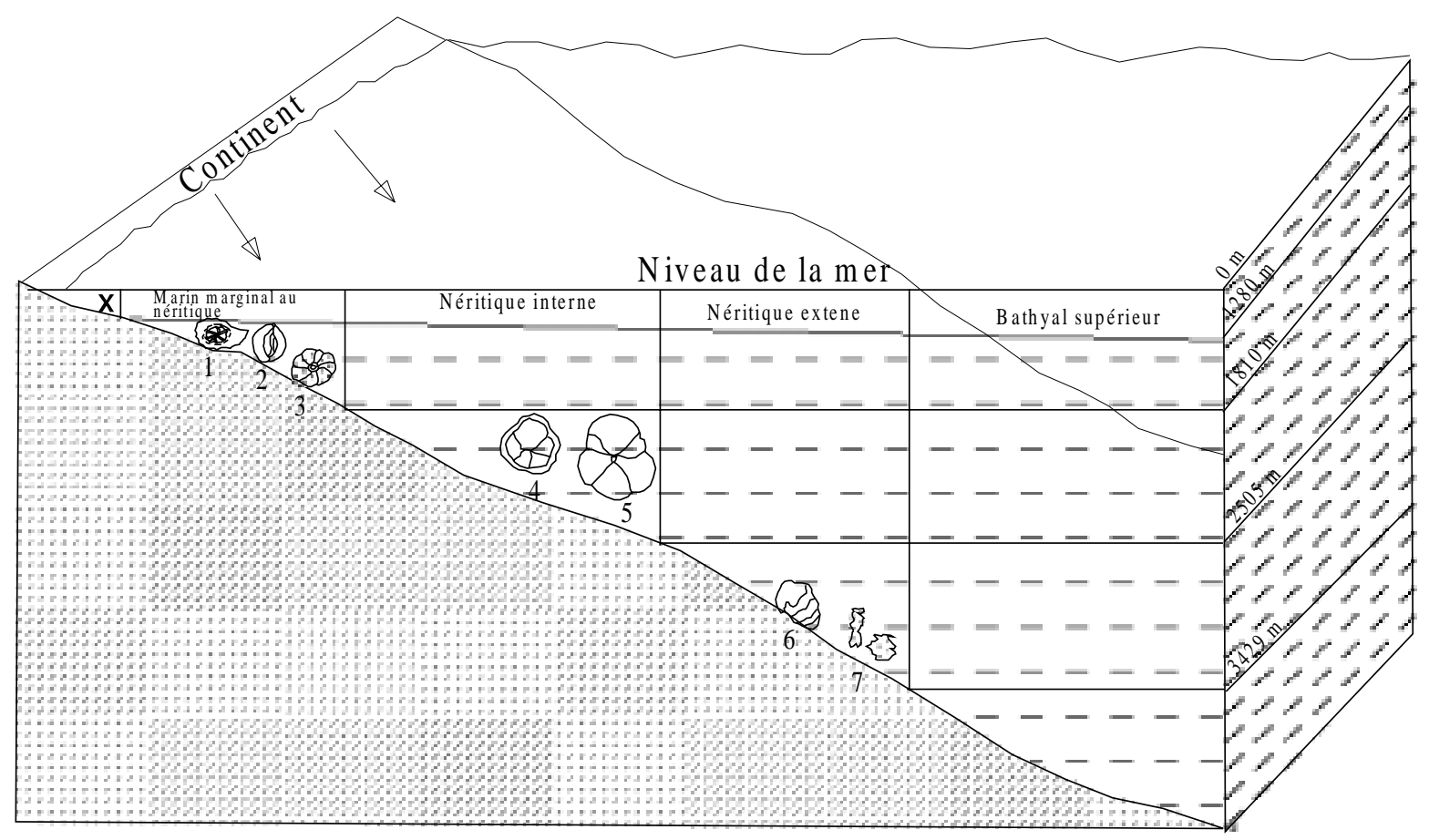

\section{LEGENDE}

\begin{tabular}{|c|l|}
\hline PALEOENVIRONNEMENT & \multicolumn{1}{|c|}{ INDICATEURS BATHYMETRIQUES } \\
\hline $\mathrm{X}=$ Lagones continentaux et marshes & \\
\hline Marin marginal au néritique interne & $\begin{array}{l}\text { Lenticulina inornata (1), Marginulina costata, } \\
\text { Ammonia beccarii, Quinqueloculina (2) }\end{array}$ \\
\hline Néritique moyen au néritique externe & $\begin{array}{l}\text { Globorotalia plesiotumida (4), G. Margaritae (5), } \\
\text { Cyclammina cancellata (3) }\end{array}$ \\
\hline Néritique externe au bathyal supérieur & $\begin{array}{l}\text { Pullenia bulloides, Saccammina complanata, Valvulina } \\
\text { flexilis (6) Haplophragmoides compressa (7) }\end{array}$ \\
\hline
\end{tabular}

Figure 5: Modèle paléoenvironnemental de dépôts et répartition de quelques espèces foraminifères benthiques dans le puits Opolo -I, Delta du Niger 


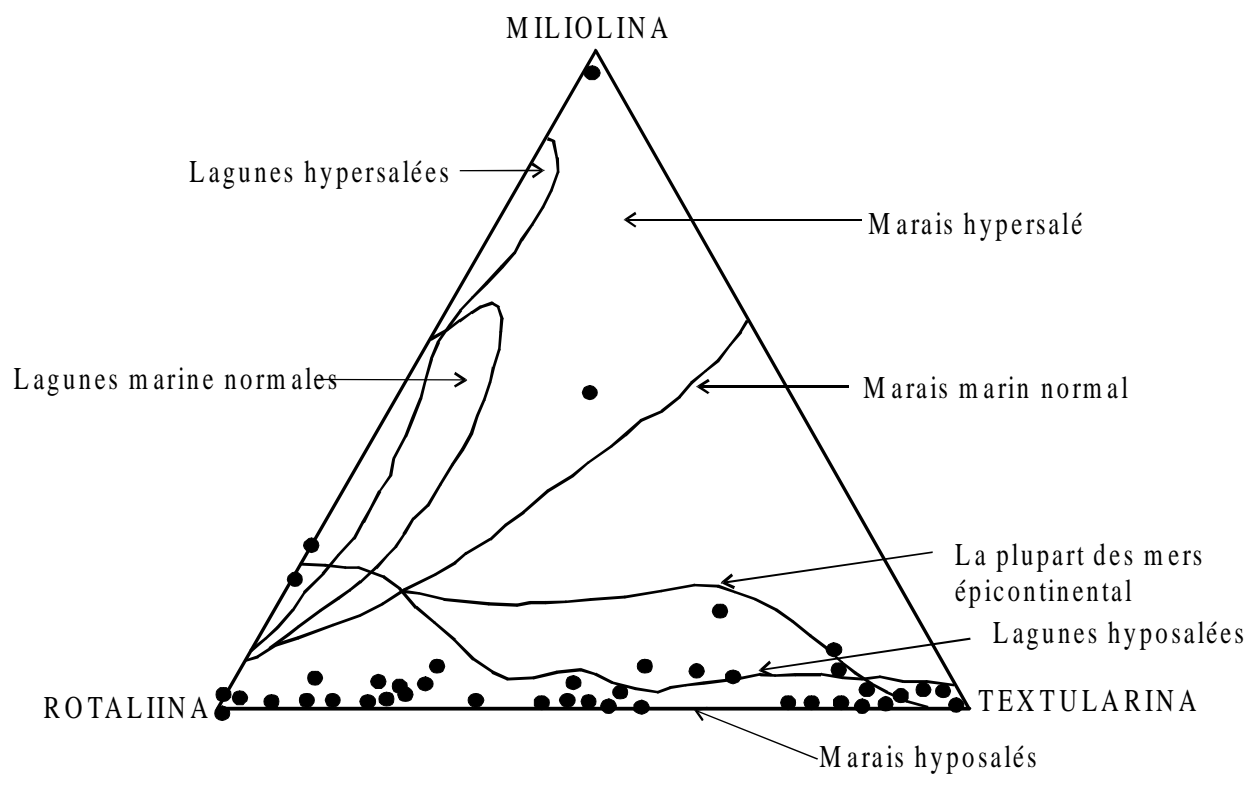

Figure 6: Rapports triangulaire de trois sous ordres des foraminifères benthiques dans le puits Opolo - I, Delta du Niger

intervalle est la présence des espèces planctiques avec une morphologie bien particuliere, c'est à dire, avec une carène caractéristique d'eau profonde (Hart et Bailey,1979a et 1979b., Hart,1980) tel que Globorotalia plesiotumida et $G$. margaritae.

\section{ENVIRONNEMENT NERITIQUE EXTERNE- BATHYAL SUPERIEUR ( 2505 - 3429 m )}

Le milieu de dépôt est indiqué par la présence de Cyclammina cancellata et Pullenia bulloides. La couleur noire des argiles très fossilifères et de la pyrite ubiquiste montrent en définitive un environnement de dépôt calme et réducteur avec peu de circulation d'eau. Cet intervalle montre donc les valeurs P/B plus élévées, avec aussi des valeurs plus élévées des espèces et la diversité. Cette augmentation remarquable en composition générique constaté ici témoigne d'une augmentation considérable du niveau de la mer provoquée par un phénomène de transgression (Mboro et al.,1981). Les assemblages de foraminifères trouvés dans cet environnement sont Saccammina complanata, Haplophragmoides compressa, Alveolophragmium crassum, Karreilla siphonella, Valvulina flexillis, Cyclammina cancellata et Pullenia bulloides. Toutes ces espèces prédominent dans les zones néritiques externes et bathyal supérieurs (Ozumba, 1995). La séquence d'Opolo-1 semble donc avoir été formée dans un bassin qui a subit un affaissement rapide variant des zones marginales peu profonde $(1280-1810 \mathrm{~m})$ pour atteindre les environnements néritique externe et bathyal supérieur en passant par la néritique moyen. L'absence de certaines zones à foraminifères (N10 à N13) montre une lacune stratigraphique.

\section{CONCLUSION}

Une étude intérgrée des sédiments provenant du puits Opolo-1dans le Delta du Niger-Nigeria, nous amene à tirer les conclusions sur les caractéristiques lithologiques, l'âge des sédiments et les paléoenvironnements de dépôts. Ainsi, on note une remarquable variation lithologique des sédiments de la base du puits ou les argiles noirs prédominent et changent progressivement en sables argileux et finallement en grès fin à grossier, très bien à mal trié au sommet du puits. Sur la base des espèces foraminifères planctiques caractéristiques récoltés de cet puits, les sédiments étaient formés pendant la tranche stratigraphique Miocène moyen - Pliocène inférieur. Les analyses biofaciés des foraminifères benthiques montrent les environnements qui varient d'une mer marginale au néritique externe et bathyal supérieur. La présence ubiquiste de la pyrite diagénétique dans toute la section étudiée est caractéristique de dépôt dans une mer calme et réductrice. 


\section{RÉMERCIEMENTS}

Les auteurs rémercient sincérement la société CHEVRON OIL COMPANY au Nigeria pour les échantillons utilisés dans cet travail.

- $\quad$ Les espèces de foraminifères récoltés dans le puits Opolo -1 sont déposées dans le Musée de Géologie de l'Université d'IBADAN, Nigeria.

\section{BIBLIOGRAPHIE CITE}

Berggren, W.A (1969): Biostratigraphy and Planktonic Foraminiferal Zonation of Tertiary System of the Sirte Basin of Libya. In P. Bronnimann, Microfossils, Geneva, 1967. Brill, Leiden: 104-120

Blow, W. H. (1969): Late Middle Eocene to Recent planktonic foraminiferal Biostratigraphy. $1^{\text {st }}$ Intern. Congr. Planktonic Microfossils, Geneva. Proceedings 1:199-422.

Bolli, H. M. (1966): The planktonic foraminifera in well Bodjonegoro-1 of Java. Eclogae Geology Helvetiae. 59: 449-465.

Cifelli, R. and Glaçon, R. (1979): New Late Miocene and Pliocene occurrences of Globorotalia species from the North Atlantic and a Paleogeographic review. Jour. Foram. Res. 9(3): 210-227

Cita, M.B. (1973): Pliocene biostratigraphy and chronostratigraphy of Sites $122 \& 124$, Leg 13. Initial Reports of DSDP 13: 1343-1379.

Hart, M.B. (1980): A water depth model for the evolution of planktonic foraminiferida. Nature, 286: 252-254

Hart, M.B. and Bailey (1979) a: The Distribution of planktonic foraminifera. Nature, 286: 252-254

Hart, M.B. and Bailey (1979) b: The Distribution of planktonic foraminifera in the Mid-Cretaceous of Northwest Europe-In: A spekte der Hreinda Europas, International Union of Geological Sciences, A, 6: 527 542.
Keller, G. (1979): Late Neogene Planktonic Foraminiferal biostratigraphy and paleo-oceanography of the Northeast Pacific Front. Jour. Foram. Res. 8:332-345.

Massala, A; Bellier, J-P; Magniez-Jannin, F. and Laurin, B. (1996): Biostratigraphy (Planktonic Foraminifera) and Environments of the Upper Cretaceous from two wells in the offshore Congo Basin; Gèologie de l' Afrique et de 1'Atlantique Sud: Actes Colloques Angers 1994:29-38.

Mboro, R., Anglada, R. and Brun, L. (1981): Le Bassin de Pointe-Noire (Congo), du Sénonien Supérieur au Néogéne (stratigraphie-paléogéographie). Cahiers de micropaléontologie 4: 73-102.

Murray, J.W. (1973): Distribution and Ecology of living foraminiferids. Heinemann, London England. 274 pp.

Ozumba, M. B (1995): Late Miocene-Pliocene biostratigraphy, offshore Niger Delta, NAPE Bull. 10: 40-48.

Parker, F.L. (1973): Late Cenozoic biostratigraphy: Planktonic foraminifera of Tropical Atlantic deep-sea sediments. Rev. Espanola Micropal. 5(2): 203-289

Petters, S. W. (1978): Mid- Cretaceous paleoenvironments and biostratigraphy of the Benue Trough, Nigeria. Geol. Soc. Am. Bull. 89: 151-154.

Postman, J.A. (1971): Manual of Planktonic foraminifera. Amsterdam, Eleven Publishing Company. 1-420.

Short, K.C. and Stauble, A. J. (1967): Outline of Geology of Niger Delta.AAPG Bull.5:65-70

Stainforth, R.M, Lamb, J.L. Luterbacher, H; Beard, J.H, and Jeffords, R.M. (1975): Cenozoic planktonic foraminiferal zonation and characteristics of index forms. Univ. of Kansas Paleontological contributions, volume 62:425pp.

Weber, K.J. (1971): Sedimentological aspects of oil fields in the Niger Delta. Geologie en Mijnbouw. Vol. 50: $559-576$. 